\title{
Schlaganfall - ein interdisziplinärer Diskurs
}

$\mathrm{D}$ er Schlaganfall hat sich in den letzten Jahren vom Stiefkind der Medizin zu einer Erkrankung entwickelt, für die wichtige, nicht-invasive diagnostische Methoden entwickelt und effektive Behandlungsund Prophylaxeverfahren erarbeitet und etabliert wurden.

Dabei hat sich das alte Basiswissen, dass neurologische Symptome zwar lokalisations- aber niemals ätiologiespezifisch sind, als unerlässliche Leitlinie erweisen. Anamnese und klinische Symptomatik erlauben dem Arzt keine sichere klinische Diagnose. So ist es nicht möglich, sicher zwischen ischämischem Insult und Hirnblutung zu differenzieren. Gleiches gilt für anamnestische Daten, wie den charakteristischen Kopfschmerz der Subarachnoidalblutung, der auch Initialsymptom einer Hirnvenenthrombose sein kann oder als Kohabitationskopfschmerz ungeklärter Genese vorkommt. Der Arzt ist daher vor jeder spezifischen Therapieentscheidung auf die modernen diagnostischen Methoden angewiesen. Unter ihnen spielen die bildgebenden Verfahren im Akutstadium die wichtigste Rolle. Unmittelbare Verfügbarkeit von CT- und MRT ist Grundvoraussetzung jeder Schlaganfallbehandlung. Dabei ist es für jeden behandelnden Arzt essentiell, Grundkenntnisse der Technik und vor allem der Aussagefähigkeit und -grenzen der Methoden zu kennen. Nur dann ist er in der Lage, das optimale Verfahren zu wählen und Diagnoseversäumnisse, Fehldiagnosen und unnötige Kosten zu vermeiden.

Der Schlaganfall ist ein akuter Notfall, für dessen diagnostische Abklärung und die daraus folgende Behandlung jede Minute zählt. Dies gilt nicht nur für die Thrombolyse beim ischämischen Insult, sondern auch für die Behandlung von Hirnblutungen, Subarachnoidalblutungen und Hirnvenenthrombosen. All diesen Krankheiten ist gemein, dass sie ein hohes Verschlechterungspotential im Frühstadium haben und der betroffene Patient durch eine Vielzahl von Komplikationen gefährdet ist.

Das Konzept der Stroke Units, auf denen Schlaganfallpatienten von spezialisierten Teams unter intermediären intensivmedizinischen Bedingungen betreut werde, trägt diesen Gesichtspunkten Rechnung.

Leider erreichen viele Patienten eine Stroke Unit nicht oder erst, wenn der optimale Zeitpunkt für Interventionen schon verstrichen ist. Dies liegt zum einen an den Patienten selber, die ihre Symptome nicht als Folge eines Schlaganfalls verstehen und in der Hoffnung auf spontane Besserung zunächst einmal abwarten. Ein zweiter Grund ist die Wahl des falschen Rettungsweges: das Warten auf den Bereitschaftsdienst oder der Besuch beim Hausarzt kosten Zeit und therapeutische Chancen für die Restitution. In einer eigenen Untersuchung zeigte sich, dass die Patienten, die unmittelbar den Notruf 112 des Rettungsdienstes wählten, die kürzesten Verzögerungszeiten hatten.
Der Rettungsorganisation in der prähospitalen Phase kommt somit entscheidende Bedeutung zu. Neben der Aufklärung der Öffentlichkeit über Grundtatsachen des Schlaganfalls haben Organisationsverbesserungen der Rettungsdienste Fortschritte gemacht. So werden z.B. vom Bereitschaftsdienst der kassenärztlichen Vereinigung in Berlin bei Notrufen regelmäßig Schlaganfallsymptome gezielt erfragt und bei Verdacht auf Schlaganfall sofortige Einlieferung per Rettungsdienst angeraten. Ein ähnliches Verfahren praktizieren viele Hausärzte.

Die Zeiten, in denen Schlaganfallpatienten Stunden auf dem Flur warten mussten, sind glücklicherweise vorbei. Dies ändert aber nichts daran, dass die Logistik in der Aufnahme von Krankenhäusern nicht verbesserungsfähig wäre. In gut organisierten Krankenhäusern mit Stroke Units sollten Zielzeiten von < 10 Minuten von Aufnahme bis erstem Arztkontakt, von 25 Min. bis zum Beginn der CT-Untersuchung und $60 \mathrm{Min}$. bis zum Therapiebeginn die Regel sein.

Im vorliegenden Heft werden in erster Linie klinische Symptomatik, Diagnose, Therapie und Prophylaxe ischämischer Schlaganfälle abgehandelt. Wegen der in den letzten Jahren erheblich gewachsenen Kenntnisse über Epidemiologie, Klinik und Prognose zerebraler Gefäßmalformationen und der immer spezieller werdenden therapeutischen Möglichkeiten ist den zerebralen Gefäßmissbildungen ein spezielles Kapitel gewidmet. Die Vielfalt der klinischen Symptomatik, die notwendigen Strategien für effektive und Kosten sparende Diagnostik, Therapie und Prophylaxe erfordern nicht nur neurologische, sondern auch internistische und allgemeinmedizinische Kenntnisse. Der Schlaganfall ist ein interdisziplinäres Problem, das eine enge Kooperation von Neurologen, Neuroradiologen, Internisten, Neurochirurgen und Gefäßchirurgen erfordert. Unglücklicherweise geschieht das meist auf dem Wege eines Konsildienstes, der eher einer Weitergabe der Verantwortung als einem interdisziplinären Diskurs gleicht. Gerade Letzterer aber ist das beste Mittel, optimale Lösungen für einen Patienten zu finden. Das vorliegende Heft möchte deswegen nicht nur dazu beitragen, die eigenen Kenntnisse zu erweitern, sondern den Neurologen auch für das Gespräch mit den Vertretern der Nachbardisziplinen zu ermuntern.

Mein Dank gilt den Koautoren, die sich mit ihren kompetenten Beiträgen dem Ziel dieses Heftes angeschlossen haben.

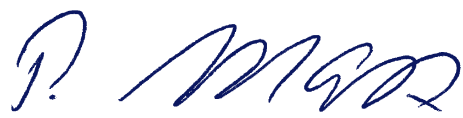

Prof. Dr. Peter Marx, Berlin 